\title{
BOUNDED COMMON EXTENSIONS OF CHARGES
}

\author{
A. BASILE, K. P. S. BHASKARA RAO, AND R. M. SHORTT
}

(Communicated by Andrew M. Bruckner)

\begin{abstract}
Let $\mathscr{A}$ and $\mathscr{B}$ be fields of subsets of a set $X$ and let $\mu: \mathscr{A} \rightarrow$ $\mathbf{R}$ and $\nu: \mathscr{B} \rightarrow \mathbf{R}$ be consistent, bounded, finitely additive measures (i.e., charges). We give necessary and sufficient conditions for $\mu$ and $\nu$ to have a bounded common extension to $\mathscr{A} \vee \mathscr{B}$. Conditions on $\mathscr{A}$ and $\mathscr{B}$ are given under which any bounded consistent charges $\mu$ and $\nu$ have a bounded common extension.
\end{abstract}

\section{INTRODUCTION}

Let $\mathscr{A}$ be a field of subsets of a set $X$. We denote by $F(X, \mathscr{A})=F(\mathscr{A})$ the linear space spanned by indicator functions $I_{A}$ of sets $A \in \mathscr{A}$. The functions in $F(X, \mathscr{A})$ have finite range and are therefore bounded. If $f \in F(X, \mathscr{A})$, then $\|f\|$ is the supremum norm of $f$.

A charge is a finitely additive set function $\mu: \mathscr{A} \rightarrow \mathbf{R}$, i.e., $\mu\left(A_{1} \cup A_{2}\right)=$ $\mu\left(A_{1}\right)+\mu\left(A_{2}\right)$ whenever $A_{1}, A_{2} \in \mathscr{A}$ are disjoint. If $\mu$ is a charge, let $|\mu|$ denote the corresponding total variation and let $\|\mu\|=|\mu|(X)$ be the total variation norm of $\mu$. A charge $\mu$ is bounded if $\|\mu\|<\infty$. A charge $\mu$ taking only the values 0 and 1 is a $0-1$ charge.

Now $(F(X, \mathscr{A}),\|\cdot\|)$ is a normed linear space whose dual may be identified with $(\mathrm{ba}(X, \mathscr{A}),\|\cdot\|)$, the space of all bounded charges on $\mathscr{A}$. Each bounded charge $\mu$ induces a linear functional $f \rightarrow \int f d \mu$ on $F(\mathscr{A})$ whose norm is $\|\mu\|$. For these and other facts about charges, we refer the reader to [1].

Let $\mathscr{A}$ and $\mathscr{B}$ be fields of subsets of a set $X$ and let $\mu, \nu$ be charges on $\mathscr{A}, \mathscr{B}$, respectively. Say that $\mu$ and $\nu$ are consistent if $\mu(C)=\nu(C)$ for all $C \in \mathscr{A} \cap \mathscr{B}$. Let $\mathscr{A} \vee \mathscr{B}$ be the field generated by $\mathscr{A} \cup \mathscr{B}$.

0.1 Lemma. Let $\mathscr{A}$ and $\mathscr{B}$ be fields of subsets of $X$ and suppose that $\mu$ and $\nu$ are charges on $\mathscr{A}$ and $\mathscr{B}$, respectively. If $\mu$ and $\nu$ are consistent, then they have a common extension, i.e., there is a charge $\rho$ on $\mathscr{A} \vee \mathscr{B}$ such that $\rho(C)=\mu(C)$ for $C \in \mathscr{A}$ and $\rho(C)=\nu(C)$ for $C \in \mathscr{B}$.

Indication. This is a well-known result. See, e.g., Theorem 3.6.2 of [1].

When do two bounded consistent charges have a bounded common extension? By the lemma, some extension exists, but might not be bounded. This

Received by the editors May 1, 1992 and, in revised form, August 13, 1992.

1991 Mathematics Subject Classification. Primary 28A99; Secondary 26A45.

Key words and phrases. Finitely additive measure, bounded charge, common extension problem. 
problem seems to have been first studied by Lipecki; some results (in a more general context) were obtained by Schmidt and Waldschaks [3]. A solution is provided by our Theorem 1.5 below. Earlier, Lipecki [2] gave some examples to show that the answer to the question is "not always". Lipecki's positive results, using global conditions on the fields $\mathscr{A}, \mathscr{B}$, follow from our work.

\section{Chain CONDitions AND Bounded EXTENSIONS}

To explain our approach, we begin with the following definition and a few abbreviations. Call $\varnothing=C_{0} \subseteq C_{1} \subseteq C_{2} \subseteq \cdots \subseteq C_{N+1}=X$ a chain in $\mathscr{A} \cup \mathscr{B}$ (of length $N$ ) if all the $C_{i}$ 's are in $\mathscr{A} \cup \mathscr{B}$. The following elementary fact lays the groundwork for our main result. Let $\mu$ and $\nu$ be consistent charges on $\mathscr{A}$ and $\mathscr{B}$, respectively: we define $\eta$ on $\mathscr{A} \cup \mathscr{B}$ by putting $\eta(C)=\mu(C)$ if $C \in \mathscr{A}$ and $\eta(C)=\nu(C)$ if $C \in \mathscr{B}$.

1.1 Lemma. Let $\mu$ on $\mathscr{A}$ and $\nu$ on $\mathscr{B}$ be consistent bounded charges. If $\rho$ on $\mathscr{A} \vee \mathscr{B}$ is a common extension of $\mu$ and $\nu$, then for any finite chain $\varnothing=C_{0} \subseteq C_{1} \subseteq C_{2} \subseteq \cdots \subseteq C_{N+1}=X$ in $\mathscr{A} \cup \mathscr{B}$,

$$
\sum_{i=0}^{N}\left|\eta\left(C_{i+1}\right)-\eta\left(C_{i}\right)\right| \leq\|\rho\|,
$$

where $\eta$ is defined as above.

From the lemma, it follows that if $\mu$ and $\nu$ have a bounded common extension, then the supremum of the left-hand side of $(*)$, taken over all possible finite chains, must be finite. Our main result establishes the converse statement (Theorem 1.5).

Let $\mathscr{A}$ and $\mathscr{B}$ be fields of subsets of $X$ and let $\mu$ on $\mathscr{A}$ and $\nu$ on $\mathscr{B}$ be consistent bounded charges. We define

$$
\begin{gathered}
I=I(\mu, \nu)=\inf \{\|\rho\|: \rho \text { a common extension of } \mu \text { and } \nu \text { to } \mathscr{A} \vee \mathscr{B}\}, \\
S=S(\mu, \nu)=\sup \left\{\int f d \mu+\int g d \nu: f \in F(\mathscr{A}), g \in F(\mathscr{B}),\|f+g\| \leq 1\right\}, \\
S C=S C(\mu, \nu)=\sup \left\{\sum_{i=0}^{N}\left|\eta\left(C_{i+1}\right)-\eta\left(C_{i}\right)\right|: \varnothing=C_{0} \subseteq C_{1} \subseteq C_{2}\right. \\
\left.\subseteq \cdots \subseteq C_{N+1}=X \text { chain in } \mathscr{A} \cup \mathscr{B}, N \geq 0\right\} .
\end{gathered}
$$

1.2 Theorem. Let $\mathscr{A}$ and $\mathscr{B}$ be fields of subsets of a set $X$ and suppose that $\mu$ and $\nu$ are consistent charges on $\mathscr{A}$ and $\mathscr{B}$, respectively. Then $S(\mu, \nu)=$ $I(\mu, \nu)$.

The infimum defining $I=I(\mu, \nu)$ is attained at some choice of $\rho$. If $\mathscr{A}$ and $\mathscr{B}$ are finite, then the supremum defining $S=S(\mu, \nu)$ is attained for some $f$ and $g$.

Proof. Given $f \in F(\mathscr{A}), g \in F(\mathscr{B}),\|f+g\| \leq 1$, and some common extension $\rho$ of $\mu$ and $\nu$, we have

$$
\int f d \mu+\int g d \nu=\int(f+g) d \rho \leq \int\|f+g\| d|\rho| \leq\|\rho\|,
$$


so that $S \leq I$. If $S=\infty$, there is nothing to prove. If $S<\infty$, consider the linear subspace $M$ of $F(\mathscr{A} \vee \mathscr{B})$ defined by

$$
M=\{f+g: f \in F(\mathscr{A}), g \in F(\mathscr{B})\} .
$$

Let $L: M \rightarrow \mathbf{R}$ be the linear functional defined by

$$
L(f+g)=\int f d \mu+\int g d \nu .
$$

The consistency of $\mu$ and $\nu$ ensures that $L$ is well defined. In fact, $L$ is a bounded linear functional on $M$ with norm $\|L\|=S<\infty$. The HahnBanach theorem implies that $L$ may be extended to a linear functional $L_{0}$ : $F(\mathscr{A} \vee \mathscr{B}) \rightarrow \mathbf{R}$ with $\left\|L_{0}\right\|=\|L\|$. Then $\rho(C)=L_{0}\left(I_{C}\right)$ defines a charge $\rho$ on $\mathscr{A} \vee \mathscr{B}$ with $\|\rho\|=\left\|L_{0}\right\|=S$, so that $S=I$, and the infimum is attained at $\rho$.

If $\mathscr{A}$ and $\mathscr{B}$ are finite, then $F(\mathscr{A} \vee \mathscr{B})$ is a finite-dimensional vector space, and the last statement of the theorem becomes elementary. Q.E.D.

1.3 Corollary. In order that consistent bounded charges $\mu$ and $\nu$ have a bounded common extension, it is necessary and sufficient that $S(\mu, \nu)<\infty$.

The following technical lemma will be used in the proof of our main theorem.

1.4 Lemma. Let $\mathscr{A}$ be a field of subsets of $X$ and let $\mu$ be a bounded charge on $\mathscr{A}$. If $\int f d \mu=\|\mu\|$ for $f \in F(X, \mathscr{A})$ with $\|f\| \leq 1$, then

(i) $\mu \geq 0$ on subsets of $\{x: f(x)=1\}$;

(ii) $\mu \leq 0$ on subsets of $\{x: f(x)=-1\}$;

(iii) $|\mu|(\{x:-1<f(x)<1\})=0$.

Indication. The proof is easy, using the fact that if $\sum a_{i} b_{i}=\sum\left|a_{i}\right|$ for numbers $a_{i} \neq 0$ and $\left|b_{i}\right| \leq 1$, then $b_{i}=1$ if $a_{i}>0$ and $b_{i}=-1$ if $a_{i}<0$.

1.5 Theorem. Let $\mathscr{A}$ and $\mathscr{B}$ be fields of subsets of $X$ and suppose that $\mu$ and $\nu$ are consistent charges on $\mathscr{A}$ and $\mathscr{B}$, respectively. Then $S C(\mu, \nu)=I(\mu, \nu)$.

Proof. That $S C \leq I$ follows from Lemma 1.1 .

In order to prove the reverse inequality, we use Theorem 1.2. Suppose that $f_{0} \in F(\mathscr{A}), g_{0} \in F(\mathscr{B})$, such that $\left\|f_{0}+g_{0}\right\| \leq 1$ are given. We shall demonstrate that

$$
S C \geq \int f_{0} d \mu+\int g_{0} d \nu
$$

from which fact follows $S C \geq S=I$ as desired.

Let $\mathscr{A}_{0}$ (respectively $\mathscr{B}_{0}$ ) be the smallest field for which $f_{0}$ (respectively $g_{0}$ ) is measurable. The $\mathscr{A}_{0} \subseteq \mathscr{A}$ and $\mathscr{B}_{0} \subseteq \mathscr{B}$ are finite. In order to prove (**), we may assume that $f_{0}$ and $g_{0}$ have been chosen so that

$$
\int f_{0} d \mu+\int g_{0} d \nu
$$

is the supremum of $\int f d \mu+\int f g d \nu$ over all choices of $f \in F\left(\mathscr{A}_{0}\right)$ and $g \in F\left(\mathscr{B}_{0}\right)$ with $\|f+g\| \leq 1$. (We use the final sentence of Theorem 1.2.) Applying Theorem 1.2 to $\mu_{0}$ and $\nu_{0}$, the restrictions of $\mu$ and $\nu$ to $\mathscr{A}_{0}$ and 
$\mathscr{B}_{0}$, respectively, we find some common extension $\rho$ of $\mu_{0}$ and $\nu_{0}$ to $\mathscr{A}_{0} \vee \mathscr{B}_{0}$ such that

$$
\int f_{0} d \mu+\int g_{0} d \nu=\|\rho\|
$$

Hence

$$
\int\left(f_{0}+g_{0}\right) d \rho=\|\rho\|
$$

so that, by Lemma 1.4, $f_{0}+g_{0}= \pm 1(|\rho|$-a.e. $)$. By the same Lemma, $\rho \geq 0$ for subsets of $\left\{x: f_{0}(x)+g_{0}(x)=1\right\}$ and $\rho \leq 0$ for subsets of $\left\{x: f_{0}(x)+g_{0}(x)=\right.$ $-1\}$.

Now $f_{0}$ and $g_{0}$ may be replaced with $f_{0}+c$ and $g_{0}-c$ for any constant $c$, with no effect on the norm or integral of their sum. Thus, without loss of generality, we may assume that $f_{0} \geq 0, g_{0} \leq 0$. Let $N$ be an even integer such that $N \geq \max \left\{\left\|f_{0}\right\| \cdot\left\|g_{0}\right\|\right\}$ and define

$$
C_{i}= \begin{cases}\left\{x \in X: g_{0}(x) \leq-N+i-1\right\} & \text { if } i \text { is odd, } \\ \left\{x \in X: f_{0}(x) \geq N-i+1\right\} & \text { if } i \text { is even. }\end{cases}
$$

Then $\varnothing=C_{0} \subseteq C_{1} \subseteq \cdots \subseteq C_{N+1}=X$ is a chain of sets in $\mathscr{A} \cup \mathscr{B}$. If $i$ is odd, then $f_{0}+g_{0}>-1$ on $C_{i+1}-C_{i}$, so that $f_{0}+g_{0}=1|\rho|$-a.e. on $C_{i+1}-C_{i}$. Likewise, if $i$ is even, then $f_{0}+g_{0}=-1|\rho|$-a.e. on $C_{i+1}-C_{i}$.

Define functions $f_{1} \in F(\mathscr{A})$ and $g_{1} \in F(\mathscr{B})$ by putting

$$
\begin{array}{ll}
f_{1}=N-2 n-1 & \text { for } x \in C_{2 n+2}-C_{2 n}, \\
g_{1}=-N+2 n & \text { for } x \in C_{2 n+1}-C_{2 n-1}
\end{array}
$$

for $n=0,1, \ldots, N / 2$, noting that $C_{-1}=\varnothing$ and $C_{N+2}=X$. For $i$ odd, $f_{1}+g_{1}=1$ on $C_{i+1}-C_{i}$. For $i$ even, $f_{1}+g_{1}=-1$ on $C_{i+1}-C_{i}$. Thus

$$
\begin{aligned}
S C & \geq \sum_{i=0}^{N}\left|\eta\left(C_{i+1}\right)-\eta\left(C_{i}\right)\right|=\int\left(f_{1}+g_{1}\right) d \rho \\
& =\int\left(f_{0}+g_{0}\right) d \rho=\int f_{0} d \mu+\int g_{0} d \nu \text {. Q.E.D. }
\end{aligned}
$$

1.6 Corollary. In order that consistent bounded charges $\mu, \nu$ have a bounded common extension, it is necessary and sufficient that $S C(\mu, \nu)<\infty$.

Inspection of the proof of Theorem 1.5 yields the following useful sharpening of the result.

1.7 Corollary. In the supremum used to define $S C(\mu, \nu)$, it suffices to restrict attention to chains of the form $\varnothing=C_{0} \subseteq C_{1} \subseteq \cdots \subseteq C_{N+1}=X$, where $C_{i} \in \mathscr{A}$ if $i$ is even, and $C_{i} \in \mathscr{B}$ if $i$ is odd.

\section{Global CONDitions of FieldS}

Let $\mathscr{A}$ and $\mathscr{B}$ be fields of subsets of $X$. Then $\mathscr{A}$ and $\mathscr{B}$ are independent if $A \cap B \neq \varnothing$ whenever $A \in \mathscr{A}$ and $B \in \mathscr{B}$ are nonvoid sets. In particular, this implies that $\mathscr{A} \cap \mathscr{B}=\{\varnothing, X\}$. The following result is probably known (reference unknown), but now follows from the theory of the previous section. 
2.1 Theorem. Let $\mathscr{A}$ and $\mathscr{B}$ be independent fields of subsets of $X$ and suppose that $\mu$ and $\nu$ are consistent charges on $\mathscr{A}$ and $\mathscr{B}$. (Consistency means only that $\mu(X)=\nu(X)$.) Then $\mu$ and $\nu$ have a common extension $\rho$ on $\mathscr{A} \vee \mathscr{B}$ such that $\|\rho\|=\max \{\|\mu\|,\|\nu\|\}$.

Proof. We apply Theorem 1.5 and Corollary 1.7. Independence essentially limits the length of chains as in Corollary 1.7. It suffices to consider chains of the form $\varnothing \subseteq A \subseteq X$ for $A \in \mathscr{A}$ or $\varnothing \subseteq B \subseteq X$ for $B \in \mathscr{B}$. The supremum $S C(\mu, \nu)$ is thus taken over quantities of the form $|\mu(A)|+|\mu(X-A)|$ or $|\nu(B)|+|\nu(X-B)|$. The result follows. Q.E.D.

Fields $\mathscr{A}$ and $\mathscr{B}$ over $X$ are weakly independent, a notion due to Lipecki [2], if whenever $X=A_{1} \cup \cdots \cup A_{n}$ and $X=B_{1} \cup \cdots \cup B_{m}$ are partitions of $X$ into nonempty sets $A_{i} \in \mathscr{A}$ and $B_{i} \in \mathscr{B}$, then there is some $k$ and some $l$ such that $A_{k} \cap B_{i} \neq \varnothing$ (each $i$ ) and $A_{i} \cap B_{l} \neq \varnothing$ (each $i$ ). The following is a slight improvement on a result of Lipecki [2, Proposition 1].

2.2 Theorem. Let $\mathscr{A}$ and $\mathscr{B}$ be weakly independent fields of subsets of a set $X$ and suppose that $\mu$ and $\nu$ are consistent charges on $\mathscr{A}$ and $\mathscr{B}$ (this means only that $\mu(X)=\nu(X))$. Then there is a common extension $\rho$ of $\mu$ and $\nu$ such that $\|\rho\| \leq\|\mu\|+|\mu(X)|+\|\nu\|$.

Proof. Apply Theorem 1.5 and Corollary 1.7. Weak independence limits the length of the chains as in Corollary 1.7. They are either of the form $\varnothing \subseteq A \subseteq$ $B \subseteq X$ or $\varnothing \subseteq B \subseteq A \subseteq X$ for $A \in \mathscr{A}$ and $B \in \mathscr{B}$. The supremum $S C(\mu, \nu)$ is thus taken over quantities

$$
|\mu(A)|+|\nu(B)-\mu(A)|+|\nu(X)-\nu(B)|
$$

or

$$
|\nu(B)|+|\mu(A)-\nu(B)|+|\mu(X)-\mu(A)| .
$$

Both of these are bounded by

$$
|\mu(A)|+|\mu(X)-\mu(A)|+|\mu(X)|+|\nu(B)|+|\nu(X)-\nu(B)|,
$$

which quantity does not exceed $\|\mu\|+|\mu(X)|+\|\nu\|$. Q.E.D.

Let us call a chain (finite or infinite) a real chain if each difference $C_{i+1}-C_{i}$ is nonempty, and each of its sets $C_{i}$ (except for the first and last) is in exactly one of $\mathscr{A}, \mathscr{B}$, and $C_{i+1}$ is in $\mathscr{B}$ (respectively $\mathscr{A}$ ) whenever $C_{i}$ is in $\mathscr{A}$ (respectively $\mathscr{B}$ ). With reference to the preceding theorems, we note that $\mathscr{A}$ and $\mathscr{B}$ are independent if and only if every real chain in $\mathscr{A} \cup \mathscr{B}$ is of length $\leq 1$. If $\mathscr{A}$ and $\mathscr{B}$ are weakly independent, then every real chain in $\mathscr{A} \cup \mathscr{B}$ is of length $\leq 2$. This latter property is slightly weaker than weak independence, as is shown by the following example. This bound on the length of real chains $(\leq 2)$ is, however, all that was needed to prove Theorem 2.2.

2.3 Example. Set $X=\{1,2,3,4,5,6\}$, let $\mathscr{A}$ be generated by the partition $\{1,2\},\{3,4\},\{5,6\}$, and let $\mathscr{B}$ be generated by the partition $\{1,5\}$, $\{2,3\},\{4,6\}$. Then each real chain in $\mathscr{A} \cup \mathscr{B}$ is of length $\leq 2$, so that the conclusion of Theorem 2.2 applies, but $\mathscr{A}$ and $\mathscr{B}$ are not weakly independent.

2.4 Theorem. Let $\mathscr{A}$ and $\mathscr{B}$ be fields of subsets of a set $X$ such that $\mathscr{A} \cap \mathscr{B}=$ $\{\varnothing, X\}$. Consider the following statements: 
(i) All real chains in $\mathscr{A} \cup \mathscr{B}$ are of bounded length.

(ii) Any two bounded, consistent charges on $\mathscr{A}$ and $\mathscr{B}$ have a bounded common extension.

(iii) Any two consistent $0-1$ charges on $\mathscr{A}$ and $\mathscr{B}$ have a bounded common extension.

(iv) All real chains in $\mathscr{A} \cup \mathscr{B}$ are of finite length.

Then these implications obtain: (i) $\Rightarrow$ (ii) $\Rightarrow$ (iii) $\Rightarrow$ (iv). Also, (iii) does not imply (ii).

Proof. (i) $\Rightarrow$ (ii): Let $K$ be an upper bound for the length of real chains in $\mathscr{A} \cup \mathscr{B}$. Let $\mu$ and $\nu$ be bounded, consistent charges on $\mathscr{A}$ and $\mathscr{B}$ and let $\eta(C)=\mu(C)$ for $C \in \mathscr{A}$ and $\eta(C)=\nu(C)$ for $C \in \mathscr{B}$. Then for each real chain $\varnothing=C_{0} \subseteq C_{1} \subseteq \cdots \subseteq C_{N+1}=X$ we have

$$
\sum_{i=0}^{N}\left|\eta\left(C_{i+1}\right)-\eta\left(C_{i}\right)\right| \leq N(\|\mu\|+\|\nu\|) .
$$

Corollary 1.7 for the calculation of $S C(\mu, \nu)$ allows one to look only at real chains. It follows from $(* * *)$ that $S C(\mu, \nu) \leq K(\|\mu\|+\|\nu\|)<\infty$, so that $\mu$ and $\nu$ have a bounded common extension.

(ii) $\Rightarrow$ (iii): Trivial.

(iii) $\Rightarrow$ (iv): This was observed by Lipecki [2, Example 1]. If $\varnothing=C_{0} \subseteq$ $C_{1} \subseteq \cdots$ is an infinite real chain with $C_{i} \in \mathscr{A}$ for $i$ odd and $C_{i} \in \mathscr{B}$ for $i$ even, take a $0-1$ charge $\mu$ on $\mathscr{A}$ such that $\mu\left(C_{1}\right)=1$ and a $0-1$ charge $\nu$ on $\mathscr{B}$ such that $\nu\left(C_{i}\right)=0$ for $i$ even. Then $\mu$ and $\nu$ are consistent, and $S C(\mu, \nu)=\infty$, so that there is no bounded common extension.

To prove that (iii) does not imply (ii), we offer the following.

2.5 Example. Set $X=\{1,2,3,4, \ldots\}$. Let $\mathscr{A}$ be the field generated by the sets $\{2 k+1,2 k+2\}, k=0,1,2, \ldots$. Let $\mathscr{B}$ be the field generated by the singleton sets $\left\{2^{l+1}-1\right\}$ for $l=0,1, \ldots$ and the sets $\left\{2^{l+1}+2 k-2,2^{l+1}+\right.$ $2 k-1\}$ for $l=1,2, \ldots$ and $1 \leq k \leq 2^{l}-1$. Then $\mathscr{A} \cap \mathscr{B}=\{\varnothing, X\}$, all real chains in $\mathscr{A} \cup \mathscr{B}$ are of finite length, but are not of bounded length. Any two 0 -1 charges on $\mathscr{A}$ and $\mathscr{B}$ have a bounded common extension.

Define charges $\mu$ and $\nu$ on $\mathscr{A}$ and $\mathscr{B}$ as follows. Put $\mu(A)=0$ for $A \in \mathscr{A}$ finite and $\mu(A)=1$ for $A \in \mathscr{A}$ cofinite. Put $\nu\left(\left\{2^{l+1}-1\right\}\right)=2^{-l-1}$ and $\nu(\{k, k+1\})=0$ if $\{k, k+1\} \in \mathscr{B}$. Also set $\nu(X)=1$. Then $\mu$ and $\nu$ are bounded, consistent charges with no bounded common extension.

We shall conclude with an example to show that the hypothesis that $\mathscr{A} \cap \mathscr{B}=$ $\{\varnothing, X\}$ is needed in Theorem 2.4. To wit, if this is not assumed, then even if there are infinite real chains in $\mathscr{A} \cup \mathscr{B}$, it is possible that any two consistent 0-1 charges have a bounded common extension.

2.6 Example. Set $X=\{1,2,3, \ldots\}$. Let $\mathscr{A}$ be the field generated by the collection $\{1\},\{2,3\},\{4\},\{5,6\},\{7\},\{8,9\}, \ldots$ and let $\mathscr{B}$ be the field generated by $\{1,2\},\{3\},\{4,5\},\{6\},\{7,8\},\{9\}, \ldots$. Then $\mathscr{A} \cap \mathscr{B} \neq$ $\{\varnothing, X\}$, there are infinite real chains in $\mathscr{A} \cup \mathscr{B}$, but any two bounded consistent charges on $\mathscr{A}$ and $\mathscr{B}$ have a bounded common extension.

2.7 Question. With reference to Theorem 2.4, can one prove the implications (ii) $\Rightarrow$ (i) and (iv) $\Rightarrow$ (iii)? 


\section{REFERENCES}

1. K. P. S. Bhaskara Rao and M. Bhaskara Rao, Theory of charges, Academic Press, London and New York, 1983.

2. Z. Lipecki, On common extensions of two quasi-measures, Czechosovak Math. J. 36 (1986), 489-494.

3. K. D. Schmidt and G. Waldschaks, Common extensions of order bounded vector measures, Rend. Circ. Mat. Palermo (2) Suppl. 28 (1992), 117-124.

Dipartimento di Matematica e Applicazioni, Università Federico II, Naples, Italy

E-mail address: delucia@na.infn.it

Division of Statistics and Mathematics, Indian Statistical Institute, Bangalore 560059, INDIA

Current address: Department of Mathematics, Wesleyan University, Middletown, Connecticut 06459

E-mail address: isibang!kpsbrao@iisc.ernet. in

Department of Mathematics, Wesleyan University, Middletown, Connecticut 06459

E-mail address: rshortt@eagle.wesleyan.edu 